\title{
MOLECULAR DESCRIPTORS OF BENZENOID SYSTEMS
}

Nazeran Idrees ${ }^{\mathrm{a}, *}$, Muhammad Nawaz Naeem ${ }^{\mathrm{a}}$, Fida Hussain ${ }^{\mathrm{a}}$, Afshan Sadiq ${ }^{\mathrm{b}}$ and Muhammad Kamran Siddiqui

a'Department of Mathematics, Government College University Faisalabad, Faisalabad, 38000, Punjab, Pakistan

${ }^{b}$ Abdus Salam School of Mathematical Sciences, Government College University, Lahore, 54000, Punjab, Pakistan

'Department of Mathematics, Comsats Institute of Information Technology, Sahiwal, 57000, Punjab, Pakistan

Recebido em 31/05/2016; aceito em 29/08/2016; publicado na web em 29/09/2016

\begin{abstract}
Molecular descriptors are being widely used in QSAR/QSPR studies in chemistry and drug designing as well as modeling of compounds. Different topological descriptors have been formulated to investigate the physio chemical properties and chemical reactivity of compounds. In this article we gave exact relations for first and second Zagreb index, hyper Zagreb index, multiplicative Zagreb indices as well as first and second Zagreb polynomials for some benzenoid systems.
\end{abstract}

Keywords: benzenoid hydrocarbons; chemical graphs; topological indices.

\section{INTRODUCTION}

Benzenoid hydrocarbons are present naturally in our environment, minerals, food and are also produced as byproducts in certain reaction and have large scale applications in chemical industry. Apart from their extensive use, benzenoid hydrocarbons are carcinogenic and pollutants. Benzenoid systems are actually hydrogen depleted benzenoid hydrocarbons.

Benzenoid systems are planar, consist of regular hexagons which do no overlap and have no hole as shown in Figure 1.<smiles>C1CCC2CC3C(CCC4CCCCC43)CC2C1</smiles>

(a)

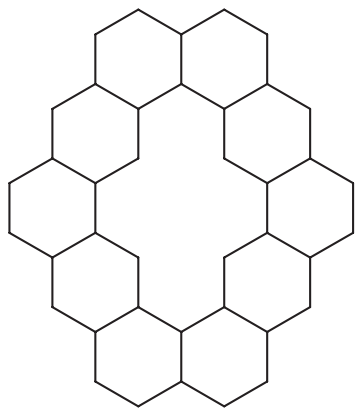

(b)
Figure 1. An example of benzenoid system(a) and a non-benzenoid system (b)

Let $G$ be a simple graph, with set of vertices $V$ and set of edges $E$. A molecular Graph is a simple connected graph where vertices denote atoms and edges denote bonds between atoms of the chemical compound. A molecular descriptor is a single numerical value which correlates the chemical structure with certain Physio chemical properties of the compound and is invariant under graph automorphisms. A large class of molecular descriptors depend on degree of vertices and are called degree based molecular descriptors. Degree of a vertex, say, $v$ is number of vertices joined to $v$ by an edge of the graph, and is denoted by $d(v)$.

Zagreb Indices are one of the oldest known topological invariants which first appeared as terms in a formula for analysis of $\pi$-electron energy and they grow with the branching of chemical graphs. ${ }^{2}$ Balaban et $a l .^{3}$ named them "Zagreb group indices" which later on termed

*e-mail: nazeranjawwad@gmail.com as first Zagreb index and second Zagreb index and are defined as:

$$
\begin{aligned}
& M_{1}(G)=\sum_{\mathrm{uv} \in \mathrm{E}(\mathrm{G})}[\operatorname{deg}(u)+\operatorname{deg}(v)] \\
& M_{2}(G)=\sum_{\mathrm{uv} \in \mathrm{E}(\mathrm{G})}[\operatorname{deg}(u) \times \operatorname{deg}(v)]
\end{aligned}
$$

In 2013, Shirdel et $a l .{ }^{4}$ introduced hyper-Zagreb index which is defined as

$$
H M(G)=\sum_{\mathrm{uv} \in \mathrm{E}(\mathrm{G})}[\operatorname{deg}(u)+\operatorname{deg}(v)]^{2}
$$

Ghorbani and Azimi defined first multiple Zagreb index $P M_{1}(G)$ and second multiple Zagreb index $P M_{2}(G)$ of a graph $G$ in $2012 .^{5}$ These are given by the following formulae:

$$
\begin{aligned}
& P M_{1}(G)=\prod_{\mathrm{uv} \in \mathrm{E}(\mathrm{G})}[\operatorname{deg}(u)+\operatorname{deg}(v)] \\
& P M_{2}(G)=\prod_{\mathrm{uv} \in \mathrm{E}(\mathrm{G})}[\operatorname{deg}(u) \times \operatorname{deg}(v)]
\end{aligned}
$$

The first Zagreb polynomial $M_{1}(G, x)$ and second Zagreb polynomial $M_{2}(G, x)$ are defined as:

$$
\begin{aligned}
& M_{1}(G, x)=\sum_{\mathrm{uv} \in \mathrm{E}(\mathrm{G})} \mathrm{x}^{[\operatorname{deg}(u)+\operatorname{deg}(v)]} \\
& M_{2}(G, x)=\sum_{\mathrm{uv} \in \mathrm{E}(\mathrm{G})} \mathrm{x}^{[\operatorname{deg}(u)+\operatorname{deg}(v)]}
\end{aligned}
$$

These new variants of Zagreb indices have been extensively studied recently. ${ }^{6-12}$

\section{RESULTS AND DISCUSSION}

\section{Zigzag benzenoid system}

Consider the graph $G$ of zigzag benzenoid system, say, $Z_{n}$ as shown in Figure 2. Here $\mathrm{n}$ denote number of rows in $Z_{n}$ and there are two hexagons in each row of the system. $Z_{n}$ has $8 n+2$ vertices and $10 n+1$ edges.

We divide edge set on the basis of degrees of endpoints of edges of the graph and also compute number of edges in each set of the partition. All vertices have degree either two or three. The edged whose endpoint vertices have degree two can be counted by analyzing the patterns for different values of $n$. One can observe that at each level we have two such edges, one upside and one downside the chain except the endpoints where we have two more edges with endpoint degree two. Thus having total $2 n+4$ edges whose endpoints have 


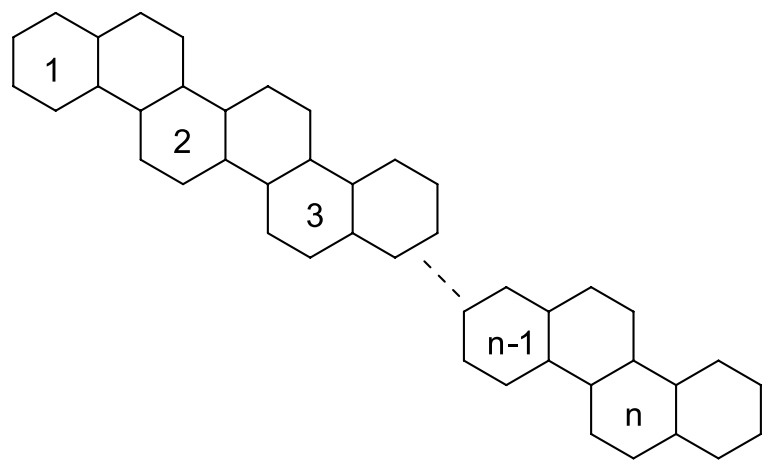

Figure 2. Graph of zigzag benzenoid system $Z_{n}$

degree $(2,2)$. Next we analyse the number of edges with endpoints degree $(2,3)$. In each row of the chain, we have four edges of this type, thus having total $4 n$ edges in a chain of length. Excluding these two type of edges from total edges, we get the edges with endpoint degrees $(3,3)$. This edge partition is summarized in Table 1 below.

Table 1. Edge partition of benzenoid graph $\mathrm{Z}_{\mathrm{n}}$ based on degree of end vertices of each edge

\begin{tabular}{lccc}
\hline$\left(d_{u}, d_{v}\right)$ where uv $\in \mathrm{E}(\mathrm{G})$ & $(2,2)$ & $(2,3)$ & $(3,3)$ \\
\hline Number of edge & $2(n+2)$ & $4 n$ & $4 n-3$ \\
\hline
\end{tabular}

We compute first Zagreb index, second Zagreb index, hyper-Zagreb index $H M(G)$, first multiple Zagreb index $P M_{1}(G)$, second multiple Zagreb index $P M_{2}(G)$, Zagreb

Polynomials $M_{1}(G, x), M_{2}(G, x)$ for $Z_{n}$ in the following theorem.

Theorem: Consider the zigzag benzenoid system $Z_{n}$, then its Zagreb are

$$
\begin{aligned}
& M_{1}(Z)=52 n-2 \\
& M_{2}\left(Z_{n}\right)=68 n-11 \\
& \left.H M_{n}\right)=308 n+20 \\
& P M_{1}\left(Z_{n}\right)=4^{2 n+4} \times 5^{4 n} \times 6^{4 n-3} \\
& P M_{2}\left(Z_{n}\right)=42^{n+4} \times 6^{4 n} \times 9^{4 n-3}
\end{aligned}
$$

Proof. Using edge partition given in Table 1, we compute the molecular descriptors defined in equations 1-5 as:

$$
\begin{aligned}
& M_{1}(G)=\sum_{u v \in E(G)}\left[d_{u}+d_{v}\right] \\
& M_{1}\left(Z_{n}\right)=\sum_{u v \in E_{1}}\left[d_{u}+d_{v}\right]+\sum_{u v \in E_{2}}\left[d_{u}+d_{v}\right]+\sum_{u v \in E_{3}}\left[d_{u}+d_{v}\right] \\
& =(2 n+4)(2+2)+(4 n)(2+3)+(4 n-3)(3+3) \\
& =52 n-2 \\
& M_{2}(G)=\sum_{u v \in E(G)}\left[d_{u} \times d_{v}\right] \\
& M_{2}\left(Z_{n}\right)=\sum_{u v \in E_{1}}\left[d_{u} \times d_{v}\right]+\sum_{u v \in E_{2}}\left[d_{u} \times d_{v}\right]+\sum_{u v \in E_{3}}\left[d_{u} \times d_{v}\right] \\
& =(2 n+4)(2 \times 2)+(4 n)(2 \times 3)+(4 n-3)(3 \times 3) \\
& =68 n-11 \\
& H M(G)=\sum_{u v \in E(G)}\left[d_{u}+d_{v}\right]^{2} \\
& H M\left(Z_{n}\right)=\sum_{u v \in E_{1}}\left[d_{u}+d_{v}\right]^{2}+\sum_{u v \in E_{2}}\left[d_{u}+d_{v}\right]^{2}+\sum_{u v \in E_{3}}\left[d_{u}+d_{v}\right]^{2} \\
& =(4 n+8)(2+2)^{2}+(4 n)(2+3)^{2}+(4 n-3)(3+3)^{2} \\
& =308 n+20
\end{aligned}
$$

$$
\begin{aligned}
& P M_{1}(G)=\prod_{u v \in E(G)}\left[d_{u}+d_{v}\right] \\
& P M_{1}\left(Z_{n}\right)=\prod_{u v \in E_{1}}\left[d_{u}+d_{v}\right] \times \prod_{u v \in E_{2}}\left[d_{u}+d_{v}\right] \times \prod_{u v \in E_{3}}\left[d_{u}+d_{v}\right] \\
& =(2+2)^{2 n+4} \times(2+3)^{4 n} \times(3+3)^{4 n-3} \\
& =(4)^{2 n+4} \times(5)^{4 n} \times(6)^{4 n-3} \\
& P M_{2}(G)=\prod_{u v \in E(G)}\left[d_{u} \times d_{v}\right] \\
& P M_{2}\left(Z_{n}\right)=\prod_{u v \in E_{1}}\left[d_{u} \times d_{v}\right] \times \prod_{u v \in E_{2}}\left[d_{u} \times d_{v}\right] \times \prod_{u v \in E_{3}}\left[d_{u} \times d_{v}\right] \\
& =(2 \times 2)^{2 n+4} \times(2 \times 3)^{4 n} \times(3 \times 3)^{4 n-3} \\
& =4^{2 n+4} \times 6^{4 n} \times 9^{4 n-3}
\end{aligned}
$$

Theorem: First Zagreb polynomial $M_{1}\left(Z_{n}, x\right)$ and second Zagreb polynomial $M_{2}\left(Z_{n}, x\right)$ of zigzag benzenoid system $Z_{n}$ are

$$
\begin{aligned}
& M_{1}\left(Z_{n} x\right)=(2 n+4) x^{4}+(4 n) x^{5}+(4 n-3) x^{6} \\
& M_{2}\left(Z_{n}, x\right)=(2 n+4) x^{4}+(4 n) x^{6}+(4 n-3) x^{9}
\end{aligned}
$$

Proof. First and second Zagreb polynomial of zigzag benzenoid chain $Z_{n}$ are computed using Table 1 as well as equations 6,7 as:

$$
\begin{aligned}
& M_{1}(G, x)=\sum_{u v \in E(G)} x^{\left[d_{u}+d_{v}\right]} \\
& M_{1}\left(Z_{n}, x\right)=\sum_{u v \in E_{1}} x^{\left[d_{u}+d_{v}\right]}+\sum_{u v \in E_{2}} x^{\left[d_{u}+d_{v}\right]}+\sum_{u v \in E_{3}} x^{\left[d_{u}+d_{v}\right]} \\
& =(2 n+4) x^{2+2}+(4 n) x^{2+3}+(4 n-3) x^{3+3} \\
& =(2 n+4) x^{4}+(4 n) x^{5}+(4 n-3) x^{6} \\
& M_{2}(G, x)=\sum_{u v \in E(G)} x^{\left[d_{u} \times d_{v}\right]} \\
& M_{2}\left(Z_{n}, x\right)=\sum_{u v \in E_{1}} x^{\left[d_{u} \times d_{v}\right]}+\sum_{u v \in E_{2}} x^{\left[d_{u} \times d_{v}\right]}+\sum_{u v \in E_{3}} x^{\left[d_{u} \times d_{v}\right]} \\
& =(2 n+4) x^{2 \times 2}+(4 n) x^{2 \times 3}+(4 n-3) x^{3 \times 3} \\
& =(2 n+4) x^{4}+(4 n) x^{6}+(4 n-3) x^{9}
\end{aligned}
$$

\section{Rhombic benzenoid system}

Consider a benzenoid system in which hexagons are arranged to form a rhombic shape, say, $R_{n}$, where $\mathrm{n}$ represents number of hexagons along each boundary of the rhomb as given in Figure 3. This benzenoid system has $2 n(n+2)$ vertices and $3 n^{2}+4 n-1$ edges. Many different benzenoid systems have been studied recently for topological invariants like trapezoid, triangular, circumcoronene and jagged rectangles.

Let $\mathrm{H}$ be the graph of zigzag benzenoid system $Z_{n}$. The edge set is partitioned into three sets, based on the degree of end vertices of each edge. The edges whose endpoints have degree $(2,2)$ appear only on the four courners of the rhomb, which are clearly 6 , whereas the edges with endpoint degree $(2,3)$ appear in pairs along the boundary of $\mathrm{H}$ except the corners where they are single. Along each boundary we have $2(n-2)+2$ edges, and summing this for four boundary lines we get total $8(n-1)$ edges, and all remaining inner edges are of type $(3,3)$. These are summarized in Table 2 below.

Table 2. Edge partition of benzenoid system $R_{n}$ based on degree of end vertices of each edge

\begin{tabular}{lccc}
\hline$\left(d_{u}, d_{v}\right)$ where uv $\in \mathrm{E}(\mathrm{G})$ & $(2,2)$ & $(2,3)$ & $(3,3)$ \\
\hline Number of edge & 6 & $8(n-1)$ & $n(3 n-4)+1$ \\
\hline
\end{tabular}




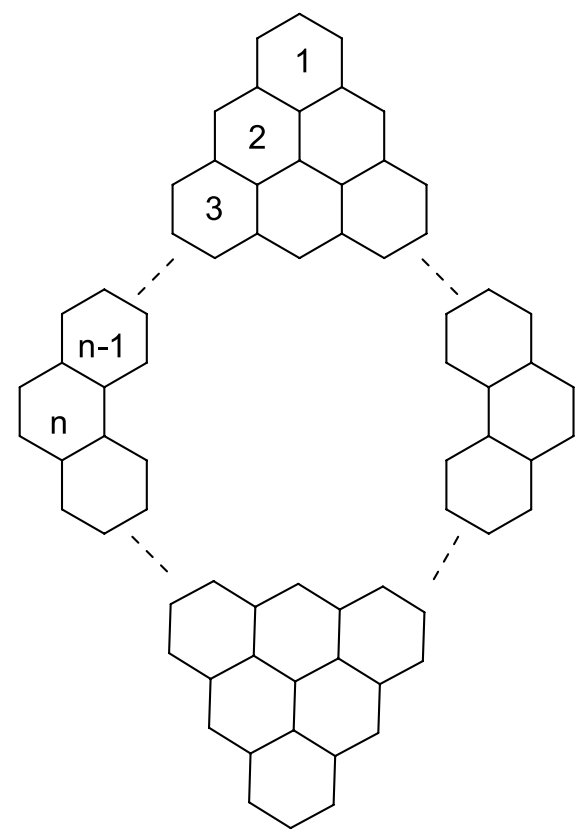

Figure 3. Graph of rhombic benzenoid system with $n$ hexagons along each boundary

In the next theorem we compute first Zagreb index $M_{1}(H)$, second Zagreb index $M_{2}(H)$, hyper-Zagreb index $H M(H)$, first multiple Zagreb index $P M_{1}(H)$, second multiple Zagreb index $P M_{2}(H)$, Zagreb polynomials $M_{1}(H, x), M_{2}(H, x)$ for $R_{n}$ in the following theorem.

Theorem: Consider the rhombic benzenoid system $R_{n}$, then its molecular descriptors are

$$
\begin{aligned}
& M_{1}\left(R_{n}\right)=18 n^{2}+16 n-10 \\
& M_{2}\left(R_{n}\right)=27 n^{2}+12 n-15 \\
& H M\left(R_{n}\right)=108 n^{2}+56 n-68 \\
& P M_{1}\left(R_{n}\right)=4^{6} \times 5^{8(n-1)} \times 6^{n(3 n-4)+1} \\
& P M_{2}\left(R_{n}\right)=4^{6} \times 6^{8(n-1)} \times 9^{n(3 n-4)+1}
\end{aligned}
$$

Proof. Using edge partition given in Table 2, we compute different variants of Zagreb indices defined in equations 1-5 as

$$
\begin{aligned}
& M_{1}(H)=\sum_{u v \in E(G)}\left[d_{u}+d_{v}\right] \\
& M_{1}\left(R_{\mathrm{n}}\right)=6(2+2)+8(n-1)(2+3)+\left(3 n^{2}-4 n+1\right)(3+3) \\
& =18 n^{2}+16 n-10 \\
& M_{2}(H)=\sum_{u v \in E(G)}\left[d_{u} \times d_{v}\right] \\
& M_{2}\left(R_{n}\right)=6(2 \times 2)+8(n-1)(2 \times 3)+\left(3 n^{2}-4 n+1\right)(3 \times 3) \\
& =27 n^{2}+12 n-15 \\
& H M(H)=\sum_{u v \in E(G)}\left[d_{u}+d_{v}\right]^{2} \\
& H M\left(R_{n}\right)=6(2+2)^{2}+8(n-1)(2+3)^{2}+\left(3 n^{2}-4 n+1\right)(3+3)^{2} \\
& =108 n^{2}+56 n-68 \\
& P M_{1}(H)=\prod_{u v \in E(G)}\left[d_{u}+d_{v}\right] \\
& P M_{1}\left(R_{n}\right)=\prod_{u v \in E_{1}}\left[d_{u}+d_{v}\right] \times \prod_{u v \in E_{2}}\left[d_{u}+d_{v}\right] \times \prod_{u v \in E_{3}}\left[d_{u}+d_{v}\right] \\
& =(2+2)^{6} \times(2+3)^{8(n-1)} \times(3+3)^{n(3 n-4)+1} \\
& =4^{6} \times 5^{8(n-1)} \times 6^{n(3 n-4)+1}
\end{aligned}
$$

$$
\begin{aligned}
& P M_{2}(H)=\prod_{u v \in E(G)}\left[d_{u} \times d_{v}\right] \\
& P M_{2}\left(R_{n}\right)=\prod_{u v \in E_{1}}\left[d_{u} \times d_{v}\right] \times \prod_{u v \in E_{2}}\left[d_{u} \times d_{v}\right] \times \prod_{u v \in E_{3}}\left[d_{u} \times d_{v}\right] \\
& =(2 \times 2)^{6} \times(2 \times 3)^{8(n-1)} \times(3 \times 3)^{n(3 n-4)+1} \\
& =4^{6} \times 6^{8(n-1)} \times 9^{n(3 n-4)+1}
\end{aligned}
$$

Theorem: First Zagreb polynomial $M_{1}\left(R_{n}, x\right)$ and second Zagreb polynomial $M_{2}\left(R_{n}, x\right)$ of rhombic benzenoid system $R_{n}$ are

$$
\begin{aligned}
& M_{1}\left(R_{n}, x\right)=6 x^{4}+8(n-1) x^{5}+\left(3 n^{2}-4 n+1\right) x^{6} \\
& M_{2}\left(R_{n}, x\right)=6 x^{4}+8(n-1) x^{6}+\left(3 n^{2}-4 n+1\right) x^{9}
\end{aligned}
$$

Proof. Now we proceed to compute the first Zagreb polynomial and second Zagreb polynomial of $R_{n}$ defined in equations 6,7 and are analyzed using Table 2 .

$$
\begin{aligned}
& M_{1}(H, x)=\sum_{u v \in E(G)} x^{\left[d_{u}+d_{v}\right]} \\
& M_{1}\left(R_{n}, x\right)=\sum_{u v \in E_{1}} x^{\left[d_{u}+d_{v}\right]}+\sum_{u v \in E_{2}} x^{\left[d_{u}+d_{v}\right]}+\sum_{u v \in E_{3}} x^{\left[d_{u}+d_{v}\right]} \\
& =6 x^{2+2}+8(n-1) x^{2+3}+\left(3 n^{2}-4 n+1\right) x^{3+3} \\
& =6 x^{4}+8(n-1) x^{5}+\left(3 n^{2}-4 n+1\right) x^{6} \\
& M_{2}(H, x)=\sum_{u v \in E(G)} x^{\left[d_{u} \times d_{v}\right]} \\
& M_{2}\left(R_{n}, x\right)=\sum_{u v \in E_{1}} x^{\left[d_{u} \times d_{v}\right]}+\sum_{u v \in E_{2}} x^{\left[d_{u} \times d_{v}\right]}+\sum_{u v \in E_{3}} x^{\left[d_{u} \times d_{v}\right]} \\
& =6 x^{2 \times 2}+8(n-1) x^{2 \times 3}+\left(3 n^{2}-4 n+1\right) x^{3 \times 3} \\
& =6 x^{4}+8(n-1) x^{6}+\left(3 n^{2}-4 n+1\right) x^{9}
\end{aligned}
$$

\section{CONCLUSIONS}

Different variants of Zagreb indices and Zagreb polynomials are analyzed for two important benzenoid systems using edge partition based on degree of vertices of the edges of the corresponding chemical graphs. We found exact relations of First Zagreb index, second Zagreb index, hyper Zagreb index, multiplicative Zagreb indices as well as Zagreb polynomials for zigzag benzenoid system and rhombic benzenoid system in above theorems, which have not been computed earlier according to the best of our knowledge.

\section{REFERENCES}

1. Gutman, I.; Cyvin, S. J.; Introduction to the Theory of Benzenoid Hydrocarbons, Springer-Verlag: Berlin, 1989.

2. Gutman, I.; Trinajstic, N.; Chem. Phys. Lett. 1972, 17, 535

3. Balaban, A. T.; Motoc, I.; Bonchev, D.; Mekenyan, O.; Topics Curr. Chem. 1983, 114, 21

4. Shirdel, G. H.; Pour, H. R.; Sayadi, A. M.; Iran. J. Math. Chem. 2013, $4,213$.

5. Ghorbani, M.; Azimi, N.; Iran. J. Math. Chem. 2012, 3, 137.

6. Eliasi, M.; Iranmanesh, A.; Gutman, I.; MATCH Commun. Math. Comput. Chem. 2012, 68, 217

7. Furtula, B.; Gutman, I.; Dehmer, M.; Appl. Math. Comput. 2013, 219, 8973.

8. Gutman, I.; Das, K. C.; MATCH Commun. Math. Comput. Chem. 2004, 52,103 .

9. Gutman, I.; Furtula, B.; Vukicevic, Z. K.; Popivoda, G.; MATCH Commun. Math. Comput. Chem. 2015, 74, 5.

10. Gutman, I.; Croat. Chem. Acta. 2013, 86, 351.

11. Hayat, S.; Imran, M.; Appl. Math. Comput. 2014, 240, 213.

12. Rada, J.; Cruz, R.; Gutman, I.; MATCH Commun. Math. Comput. Chem. 2014, 72, 125 . 